\title{
Reciclagem de Sacolas Plásticas de Polietileno em Termos de Inventário de Ciclo de Vida
}

\author{
Guilherme de C. Queiroz, Eloísa E. C. Garcia \\ Centro de Tecnologia de Embalagem, CETEA / ITAL
}

\begin{abstract}
Resumo: O CETEA realizou um estudo de Avaliação de Ciclo de Vida (ACV) de sacolas plásticas de polietileno (PE), com ênfase no inventário de ciclo de vida (ICV), coletando dados para o ano 2003. O objetivo deste artigo é apresentar parte deste estudo completo, concentrando-se na influência da taxa de reciclagem de polietileno sobre o ICV das sacolas plásticas de PE no Brasil. Para gerar os dados da média brasileira de eteno, PEAD-APM, PEAD/PELBD e PEBD, os dados foram compilados tendo em conta, respectivamente, 61, 100,78 e $63 \%$ das empresas envolvidas em cada fase do processo de produção. Depois que as empresas concordaram em colaborar com o estudo, questionários apropriados foram preparados e enviados para a coleta de dados. As empresas foram responsáveis tanto pela coleta dos dados como pelo preenchimento dos questionários. O cálculo da média brasileira da reciclagem de polietileno foi realizado considerando os dados fornecidos por sete empresas. O cálculo da média brasileira da produção das sacolas plásticas de PE foi realizado considerando os dados fornecidos por quatro empresas. Levando em conta as categorias de impacto adotadas neste estudo tem-se como resultado a demonstração de que a reciclagem ajuda a melhorar o perfil ambiental das sacolas plásticas de PE medido com dados de ICV.
\end{abstract}

Palavras-chave: Polietileno, reciclagem, inventário, ciclo de vida, sacolas.

\section{Recycling of Polyethylene Plastic Bags in Terms of Life Cycle Inventory}

\begin{abstract}
CETEA has conducted a Life Cycle Assessment (LCA) study of PE (polyethylene) plastic bags with emphasis on the Life Cycle Inventory (LCI), collecting data for the reference year 2003. The goal of this paper is to present part of this complete study, focusing on the influence of polyethylene recycling rate on the LCI of PE plastic bags in Brazil. For generating the Brazilian average data of ethylene, HDPE-HMW, HDPE/LLDPE and LDPE, the data were compiled taking into account, respectively, 61, 100, 78 and $63 \%$ of the companies involved in each phase of the production process. After the companies agreed to collaborate with the Project, appropriate questionnaires were prepared and sent for data collection. The companies were responsible for both collecting the data and completing the data sheets. The calculation of the Brazilian average polyethylene recycling was accomplished considering the data supplied by seven companies. The calculation of the Brazilian average PE plastic bags production was accomplished considering the data supplied by four companies. Taking into account the impact categories adopted in this study it has been demonstrated that recycling helps to improve the PE plastic bags environmental profile measured as LCI data.
\end{abstract}

Keywords: Polyethylene, recycling, inventory, life cycle, bags.

\section{Introdução}

O CETEA realizou um estudo de Inventário de Ciclo de Vida (ICV) de sacolas plásticas de polietileno (PE) coletando dados para o ano de referência 2003 no Brasil.

A ACV (Avaliação de Ciclo de Vida) é uma técnica para avaliar o desempenho ambiental de um determinado produto: incluindo a identificação e quantificação da energia e das matérias-primas utilizadas no seu ciclo de fabricação, bem como as emissões para a água, solo e ar inerentes da produção, utilização e disposição final, avaliando o impacto ambiental associado ao uso dos recursos naturais (energia e materiais) e emissões de poluentes e identificando oportunidades para melhorar o sistema de forma a otimizar o desempenho ambiental do produto.

O setor produtivo dos materiais plásticos já aplica a técnica de ACV para construir ICVs dos seus produtos e matérias-primas a mais de 10 anos $^{[1,2]}$. O CETEA já tem publicado diversos trabalhos de ACV nos últimos 10 anos $^{[3-8]}$. Em relação a estudos específicos de ciclo de vida de sacolas plásticas, esta é a primeira publicação com dados brasileiros. Estudos de ACV de sacolas já foram desenvolvidos nos Estados Unidos e Europa ${ }^{[9,10]}$.

Segundo a Plastivida, o Brasil reciclou aproximadamente 16\% do plástico em 2003 (aproximadamente 703 mil toneladas sendo 359 mil toneladas pós-consumo). Em 2007, esse índice foi de aproximadamente $21 \%$, que correspondeu a cerca de 962 mil toneladas de plásticos reciclado, sendo $61 \%$ pós-consumo (556 mil toneladas $)^{[11]}$.

As seguintes etapas do sistema foram inventariadas: produção de nafta, etileno, polietileno (polietileno de baixa densidade PEBD, polietileno de alta densidade - PEAD / polietileno linear de baixa densidade - PELBD, polietileno de alta densidade - alto peso molecular - PEAD-APM), produção de sacolas, reciclagem e disposição final, quantificando dados de entrada e saída como as emissões para o ar e água, os resíduos sólidos dos processos de produção e pós-consumo, a utilização de combustíveis e eletricidade, a eficiência e uso da terra.

O objetivo do estudo completo foi representar a situação brasileira média de produção e reciclagem das sacolas plásticas de PE. Alguns dos benefícios pretendidos com o desenvolvimento deste estudo foram: conhecer os parâmetros relevantes e respectivos impactos ambientais associados às sacolas plásticas de PE nas diversas etapas de seu ciclo de vida; ser um promotor de melhorias para os sistemas de produção de sacolas plásticas de PE; construir uma referência para futuras comparações, a fim de avaliar a evolução do setor; ter a informação disponível para as empresas que desejam obter rótulo ambiental dos seus produtos; avaliar os benefícios da reciclagem pós-consumo de polietileno. 
O objetivo deste trabalho é apresentar parte deste estudo completo, concentrando-se na influência da taxa de reciclagem de polietileno sobre o ICV das sacolas plásticas de PE no Brasil. A unidade funcional adotada " $1000 \mathrm{~kg}$ de sacolas plásticas de PE" se refere à quantidade de sacolas prontas para serem entregues ao mercado, mas considerando a fase pós-consumo, incluindo a coleta e reciclagem.

\section{Metodologia}

A metodologia adotada foi baseada nas normas ABNT NBR ISO 14040 e 14044 de Avaliação de Ciclo de Vida (ACV) ${ }^{[12,13]}$. O armazenamento de dados e modelagem foi realizado empregando o Software PEMS ${ }^{[14]}$.

Para gerar os dados da média brasileira de eteno, PEAD-APM, PEAD / PELBD e PEBD, os dados foram elaborados tendo em conta uma participação de, respectivamente, 61, 100, 78 e $63 \%$ das empresas envolvidas em cada fase do processo de produção, considerando-se os produtos consumidos no Brasil. Depois que a empresa concordou em colaborar com o estudo, os questionários foram preparados e enviados para a coleta de dados. As empresas foram responsáveis tanto pela coleta dos dados quanto pelo preenchimento dos questionários. $\mathrm{O}$ cálculo da média brasileira de reciclagem de polietileno foi realizado considerando os dados fornecidos por sete empresas/tecnologias. O cálculo da média brasileira de produção das sacolas plásticas de PE foi realizado considerando os dados fornecidos por quatro empresas/ tecnologias.

Somente as distâncias de transporte para entregar a média brasileira de polietileno (15\% de PEBD, 68\% de PEAD-APM e $13 \%$ de PEAD/PELBD e $4 \%$ de PEAD importado) para produzir a sacola plástica de PE "Tipo B” (no estudo completo foram também construídos cenários para uma sacola plástica de PE Tipo A com $80 \%$ de PEAD-APM e 20\% de PEAD/PELBD), a coleta seletiva da sacola plástica no pós-consumo que foi para a reciclagem ou disposição final foram considerados. Sempre que se soube que o caminhão usado para transporte de mercadorias entre a unidade de processos retornava vazio, a distância percorrida pelo caminhão carregado era contada duas vezes.

Inventários de ciclo de vida parciais, relacionados com a geração de energia elétrica, transporte rodoviário e pré-combustão, desenvolvidos pelo CETEA ${ }^{[7,15]}$, foram incorporados aos ICVs das sacolas. No caso das matérias-primas importadas, os valores médios foram coletados e completados com dados da literatura publicada ${ }^{[16]}$. As exportações não foram consideradas.

A Figura 1 ilustra a fronteira do sistema analisado no estudo completo.

O produto foi analisado a partir da extração dos recursos naturais da terra (petróleo e gás natural) até sua revalorização (reciclagem mecânica) ou disposição final.

Nove categorias de impacto foram consideradas relevantes para a situação brasileira a fim de orientar a coleta de dados: consumo de recursos naturais; mudanças climáticas (efeito estufa); acidificação; nutrificação/eutrofização; formação de fumaça fotoquímica; toxicidade humana; ecotoxicidade; utilização de volume de aterro e uso de terra.

As tintas para impressão, pigmentos, aditivos e solventes (que representam $6 \% \mathrm{p} / \mathrm{p}$ da composição das sacolas plásticas de PE) foram apenas considerados no consumo de energia e emissões associadas à sua aplicação ou processo de secagem.

A água utilizada nos processos industriais que retorna depois aos esgotos ou rios foi quantificada, enquanto que a água de sistemas fechados e/ou recirculação interna foi excluída. No entanto, a água necessária para reposição (make up) foi considerada.
Resíduos sólidos inerentes aos processos industriais foram quantificados e agregados na categoria "resíduo de processo industrial". Os materiais que foram reciclados por meio de um processo fora dos limites da fronteira do estudo (Figura 1) foram agrupados e identificados como "reciclagem externa ao sistema". Aparas internas reaproveitadas dentro da cadeia de produção, como as aparas internas de produção das sacolas plásticas de PE, foram tratadas como aproveitamento de matéria-prima (não reciclagem de aparas de conversão, conforme ABNT NBR 15792:2010 ${ }^{[17]}$ que normaliza definições e método de cálculo de índice de reciclagem de embalagem).

Finalmente, é importante notar que este estudo de ICV não inclui os investimentos de capital, ou seja, recursos e energia utilizados para a construção e manutenção dos locais de fabricação e equipamentos, estradas, usinas de energia, caminhões, e assim por diante. Apenas as entradas e saídas diretamente associadas à produção, distribuição e escoamento dos produtos em todo o seu ciclo de vida foram identificados e quantificados.

Inventários sobre a produção de $1000 \mathrm{~kg}$ de polietileno e $1000 \mathrm{~kg}$ de polietileno reciclado no pós-consumo foram construídos e, posteriormente, os inventários de ciclo de vida da produção de $1000 \mathrm{~kg}$ de sacolas plásticas de PE, considerando diferentes taxas de reciclagem, foram elaborados. A influência da taxa de reciclagem no consumo de energia e de recursos naturais e sobre os níveis de emissões são apresentados em gráficos que evidenciam a redução dos principais parâmetros inventariados. A taxa de reciclagem de $16 \%$ corresponde à percentagem de plástico reciclado em relação ao consumo interno de plásticos em 2003, enquanto $32 \%$ representam a taxa de reciclagem estimada no Brasil para o ano 2020. Todos os resultados e principais conclusões foram submetidos à análise crítica (critérios de avaliação da qualidade dos dados).

\section{Resultados e Discussão}

A Figura 2 apresenta o gráfico que ilustra a redução no consumo de recursos naturais na cadeia de produção e uso das sacolas plásticas de PE em função do aumento da taxa de reciclagem de plástico.

A redução no consumo de recursos naturais como petróleo, gás natural, água e carvão / urânio (consumidos para gerar eletricidade no sistema público brasileiro) torna-se mais expressiva com o aumento da taxa de reciclagem. A menor redução (\%) no consumo de água, em relação ao petróleo e gás natural, é devido à contribuição do processo de produção de plástico reciclado que consome água, bem como a menor redução (\%) do consumo de carvão / urânio é devido ao consumo de energia elétrica pública nas etapas de produção de sacolas plásticas e de plástico reciclado pós-consumo.

A Figura 3 apresenta o gráfico que ilustra a redução no consumo de energia na cadeia de produção e uso das sacolas plásticas de PE em função do aumento da taxa de reciclagem de plástico.

A redução no consumo de eletricidade proveniente do sistema público brasileiro não acompanhou, proporcionalmente, o aumento da taxa de reciclagem, uma vez que a energia elétrica é em grande parte consumida no processo de reciclagem do plástico e produção de sacolas plásticas.

A Figura 4 apresenta o gráfico que ilustra a redução nas emissões para o ar na cadeia de produção e uso das sacolas plásticas de PE em função do aumento da taxa de reciclagem de plástico.

Tendo em conta as emissões para o ar (ácidos, amônia, dióxido de carbono - $\mathrm{CO}_{2}$ - não renovável, hidrocarbonetos - $\mathrm{HC}$, metais, metano $-\mathrm{CH}_{4}$, monóxido de carbono - $\mathrm{CO}$, dióxido de enxofre $\mathrm{SO}_{2}$, dióxido de nitrogênio $-\mathrm{NO}_{2}$ e particulados), em geral a redução das emissões foi proporcional ao aumento da taxa de reciclagem.

As exceções foram as emissões de compostos orgânicos voláteis $(\mathrm{COV})$, devido à quantidade de solventes presentes no processo de 


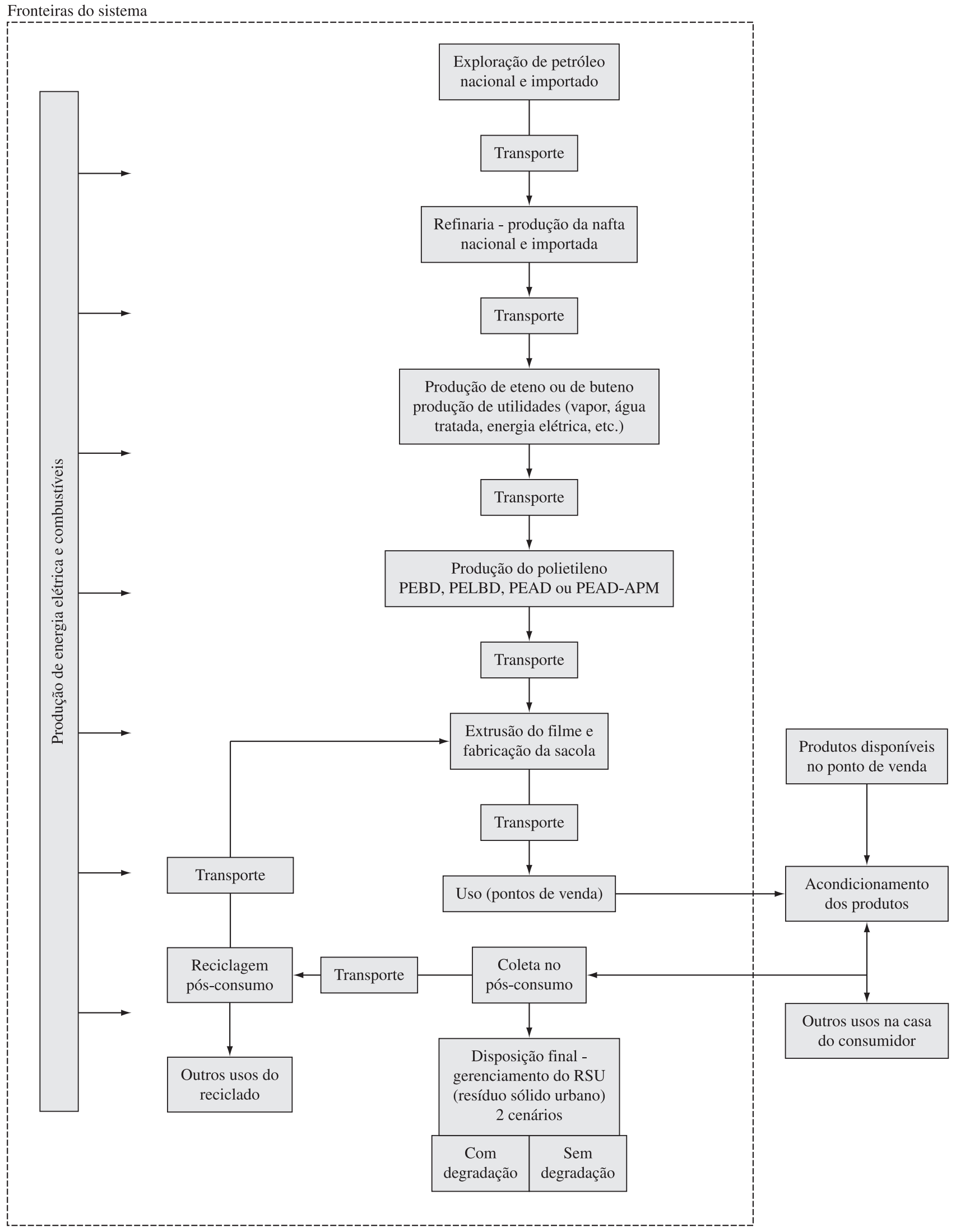

Figura 1. Fronteira do sistema analisado. 


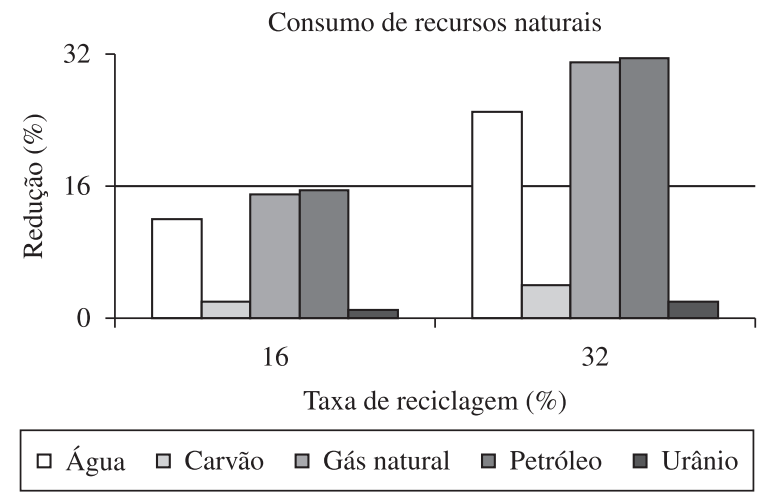

Figura 2. Redução no consumo de recursos naturais na cadeia de produção e uso de sacolas plásticas de PE, em função do aumento da taxa de reciclagem pós-consumo dos plásticos.

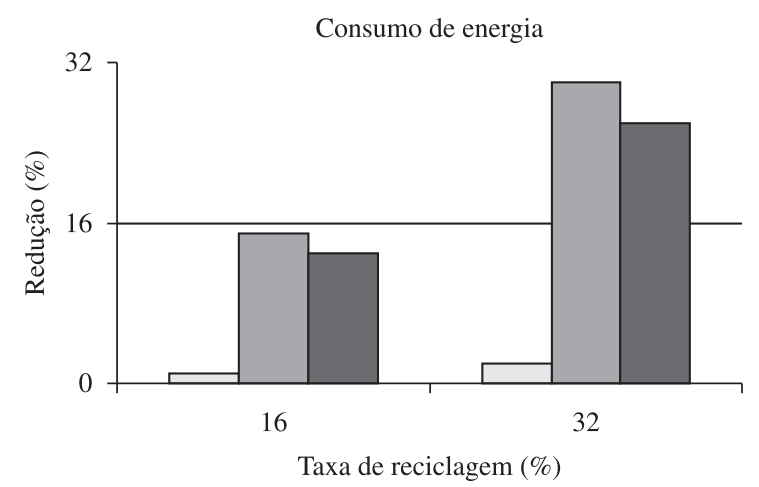

$$
\begin{aligned}
& \text { Hidreletricidade } \\
& \square \text { Energia fóssil (eletricidade e combustível) } \\
& \square \text { Energia total }
\end{aligned}
$$

Figura 3. Redução no consumo de energia na cadeia de produção e uso de sacolas plásticas de PE, em função do aumento da taxa de reciclagem pós-consumo dos plásticos.

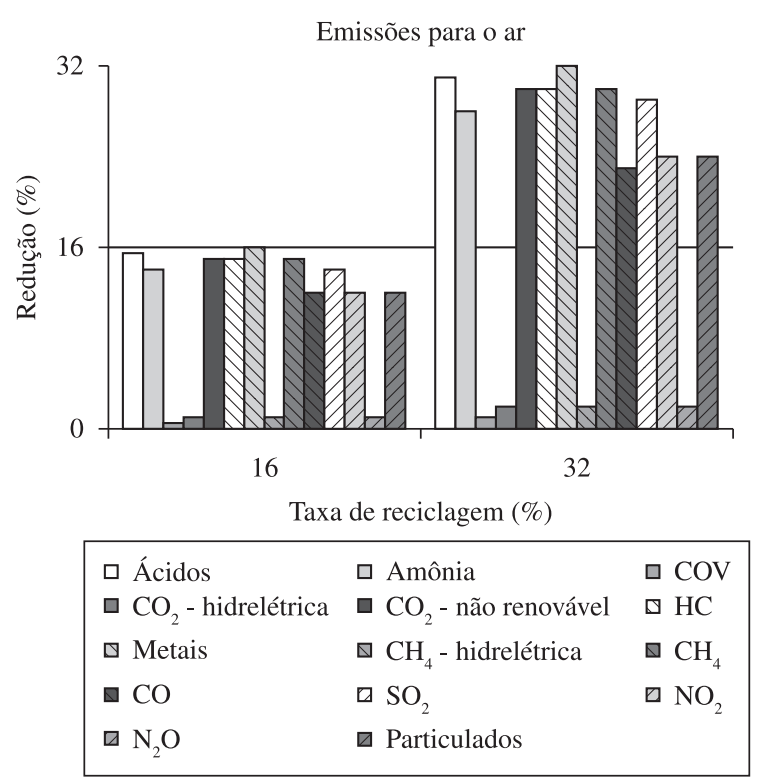

Figura 4. Redução nas emissões para o ar na cadeia de produção e uso de sacolas plásticas de PE, em função do aumento da taxa de reciclagem pós-consumo dos plásticos. impressão de sacolas plásticas, e as emissões de $\mathrm{N}_{2} \mathrm{O}$ (óxido nitroso, a partir de termelétricas a carvão) e $\mathrm{CO}_{2} / \mathrm{CH}_{4}$ (dióxido de carbono e metano das hidrelétricas) da eletricidade proveniente do sistema público brasileiro, consumidos no processo de reciclagem do plástico e produção de sacolas plásticas.

A Figura 5 apresenta o gráfico que ilustra a redução (e aumento) nas emissões para a água na cadeia de produção e uso das sacolas plásticas de PE em função do aumento da taxa de reciclagem de plástico.

Levando em conta as emissões de água (ácidos, compostos nitrogenados, compostos sulfurados, demanda bioquímica de oxigênio - DBO, demanda química de oxigênio - DQO, hidrocarbonetos - HC, fosfatos, metais, óleos e gorduras, sólidos solúveis e substâncias orgânicas dissolvidas, a redução das emissões, em geral, foi proporcional ao aumento da taxa de reciclagem. A exceção foi a emissão de "sólidos não solúveis (SNS)" devido à "lavagem" durante o processo de reciclagem.

É importante ressaltar que parte (50\%) dos resíduos do processo de "lavagem" da reciclagem foram contabilizados como "sólidos não solúveis" para a água (SNS). A outra parte (50\%) dos resíduos do processo de "lavagem" da reciclagem foi contabilizada como "resíduo de processo industrial".

A Figura 6 apresenta o gráfico que ilustra a redução de resíduos sólidos na cadeia de produção e uso das sacolas plásticas de PE em função do aumento da taxa de reciclagem de plástico.

Levando em conta os resíduos sólidos, a redução das emissões, em geral, foi proporcional ao aumento da taxa de reciclagem. A exceção foi a emissão de "resíduo de processo industrial" devido à "lavagem" durante o processo de reciclagem (considerando $50 \%$ como resíduo de processo industrial e $50 \%$ como SNS para a água).

De forma geral, é possível observar que, no caso do polietileno e dos produtos transformados de plástico, como as sacolas plásticas de $\mathrm{PE}$, os recursos naturais mais preservados pela reciclagem são o petróleo / gás natural (recursos não-renováveis) e água. Os recursos naturais utilizados para fins energéticos, até a fase de produção de polietileno também são baseados no petróleo e gás natural e por isso são os itens de maior redução no consumo quando o material é reciclado.

As emissões para a água apresentados nos inventários não permitem muitas conclusões em relação ao seu efeito específico sobre o meio ambiente, uma vez que foram reportadas principalmente em grupos (DBO, DQO, sólidos suspensos...). No entanto, elas contribuem em categorias de impacto como ecotoxicidade e eutrofização.

Parte das emissões para o ar foi quantificada por meio de cálculos estequiométricos ou fatores de emissão. Isso foi necessário nos casos em que essas emissões não foram informadas pelas empresas. Portanto, os dados dos inventários podem ser aperfeiçoados em estudos futuros, ainda mais substituindo esses valores por níveis de emissão reais.

Para os produtos transformados de plástico nem sempre a taxa de reciclagem afeta os valores de seus ICVs na mesma proporção, uma vez que a contribuição de etapas do ciclo de vida de produção da sacola e rendimento do processo de reciclagem permaneça inalterada.

Em geral, os dados não coletados sempre foram estimados tendo em conta a informação disponível, a fim de minimizar a ocorrência de falta de dados.

A colaboração das empresas foi excepcional. Neste aspecto, o estudo foi bastante diferente da grande maioria deste tipo de estudo em que a principal dificuldade é a participação do setor produtivo, uma vez que existe certo receio com a divulgação dos dados, sigilo e complexidade. Neste contexto, os objetivos do estudo foram considerados alcançados com os dados disponibilizados. 


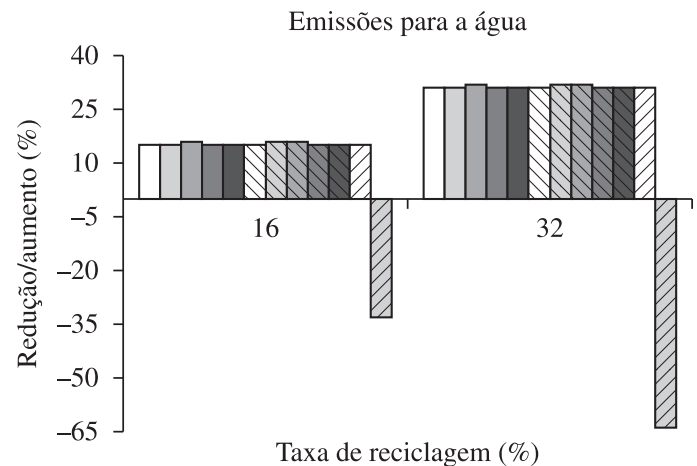

Taxa de reciclagem $(\%)$

$\square$ Ácidos
$\square$ Compostos nitrogenados
$\square$ Compostos sulfurados
$\square$ DBO
$\square$ DQO
$\square$ HC
$\square$ Fosfatos
$\square$ Metais
$\square$ Óleos e gorduras
■ Sólidos solúveis
$\square$ Substâncias orgânicas dissolvidas
$\square$ Sólidos não solúveis

Figura 5. Redução (e aumento) nas emissões para a água na cadeia de produção e uso de sacolas plásticas de PE, em função do aumento da taxa de reciclagem pós-consumo dos plásticos.

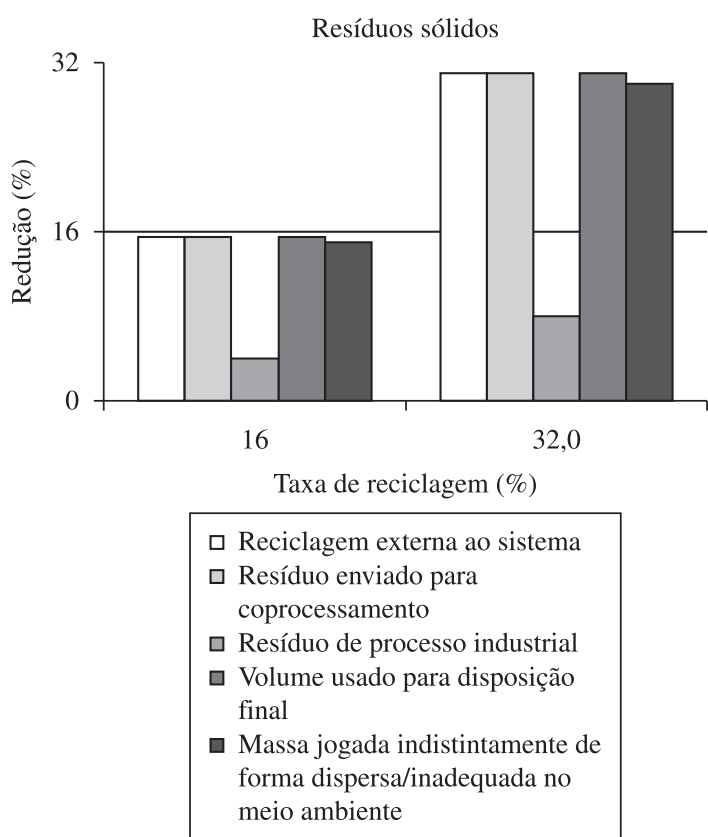

Figura 6. Redução de resíduos sólidos na cadeia de produção e uso de sacolas plásticas de PE, em função do aumento da taxa de reciclagem pós-consumo dos plásticos.

\section{Conclusões}

A representatividade dos dados deste estudo foi de 100, 78 e $63 \%$ da produção brasileira de PEAD-APM, PEAD/PELBD e PEBD, respectivamente, evidenciando uma ótima cooperação industrial com o estudo.
Para as etapas de reciclagem de plástico no pós-consumo e produção de sacolas plásticas não foi possível chegar a uma alta representatividade, devido ao grande número dessas indústrias no Brasil. Portanto, os dados foram coletados em 7 e 4 empresas destes setores, respectivamente.

A influência da taxa de reciclagem de plástico sobre os parâmetros inventariados é bastante proporcional, sendo que os indicadores de consumo e emissões diminuem à medida que aumenta a taxa de reciclagem. Este fato é esperado, pois a produção de polietileno predomina sobre quase todas as outras etapas do ciclo de vida das sacolas plásticas de PE. As vantagens da reciclagem estão, obviamente, concentradas sobre os indicadores relacionados com a produção de polietileno e volume usado para disposição final das embalagens no pós-consumo.

Devido às contribuições relativas de outras etapas do ciclo de vida, como as etapas de produção das sacolas plásticas de PE e de reciclagem do plástico pós-consumo, o aumento da taxa de reciclagem não tem a mesma magnitude em alguns indicadores inventariados. De acordo com os resultados apresentados é possível concluir que a reciclagem de plástico reduz parte do consumo de recursos naturais e energia e das emissões associadas às fases anteriores à produção da embalagem, ou seja, da produção dos polietilenos. Desta forma, conclui-se que todos os esforços feitos para aumentar a taxa de reciclagem terão uma contribuição positiva para a melhoria contínua do desempenho ambiental das sacolas plásticas.

O objetivo da melhoria contínua com foco no desempenho ambiental é desenvolver produtos com menores exigências de energia e recursos naturais e com os níveis mais baixos de emissões possíveis. Assim, o impacto ambiental será menor e mais recursos serão preservados para as gerações futuras. Neste contexto, sempre é possível melhorar!

Vale ressaltar que estudos de ICV são interativos e dinâmicos. Os dados podem sempre ser refinados, substituídos ou complementados com informação mais atualizada, representando cada vez melhor o desempenho ambiental do setor analisado. As conclusões e recomendações de um ICV são unicamente aplicadas à situação analisada, portanto, qualquer adaptação das conclusões para, por exemplo, outro país com diferente nível tecnológico e requisitos legais seria vago e questionável.

Este estudo forneceu informações básicas de ICV e modelos que permitem a posterior avaliação e interpretação de determinadas situações ambientais do setor de polietileno no Brasil.

A partir deste estudo, o setor de polietileno no Brasil é capaz de quantificar os benefícios das futuras ações de melhoria ambiental da indústria de plástico brasileira, bem como contribuir tecnicamente para o desenvolvimento de produto "sacola plástica de PE" com menor impacto ambiental.

\section{Agradecimentos}

Os autores gostariam de agradecer o apoio prestado pelo Conselho Nacional de Desenvolvimento Científico e Tecnológico - CNPq e Instituto Sócio-Ambiental dos Plásticos - Plastivida, Brasil.

\section{Referências Bibliográficas}

1. Eco-Profiles of the European Plastics Industry. - "Hydrocarbon raw materials", Association of Plastics Manufacturers in Europe - APME, Brussels (2003).

2. Eco-Profiles of the European Plastics Industry. - "Polyolefins", Association of Plastics Manufacturers in Europe - APME, Brussels (2003). 
3. Garcia, E. E. C. \& Coltro, L. - "Reciclagem de Embalagens de PET no Brasil: uma visão segundo a metodologia de análise do ciclo de vida - ACV", in: Anais do $6^{\circ}$ Congresso Brasileiro de Polímeros, Gramado (2001).

4. Gatti, J. B.; Queiroz, G. C. \& Garcia, E. E. C. - Int. J. Life Cycle Ass., 13, p.219-225 (2008).

5. Mourad, A. L.; Garcia, E. E. C.; Vilela, G. B. \& Zuben, F. V. - Resour. Conserv. Recycl., 52, p.678 (2008).

6. Mourad, A. L.; Garcia, E. E. C.; Vilela, G. B. \& Zuben, F. V. - Int. J. Life Cycle Ass., 13, p.140 (2008).

7. Coltro, L.; Garcia, E. E. C. \& Queiroz, G. C. - Int. J. Life Cycle Ass., 8, p.290 (2003).

8. Coltro, L.; Garcia, E. E. C. et al. - "Life cycle assessment of packaging systems for the Brazilian market”, in: Proceedings of R'99 - Recovery, Recycling, Re-Integration, Geneva (1999).

9. Queiroz, G. C. \& Garcia, E. E. C. - Informativo CETEA/ITAL, 20, 5p. (2008).

10. ECOBILAN. - "Évaluacion des impacts environnementaux des sacs de caisse Carrefour", (2004).

11. Plastivida - Instituto Sócio-Ambiental dos Plásticos. - "Monitoramento dos índices de reciclagem mecânica dos plásticos no Brasil” Disponível em: <http://www.plastivida.org.br/2009/pdfs/IndiceReciclagem2007. pdf>. Acesso em: 27 jan. 2010.

12. Associação Brasileira de Normas Técnicas. - "NBR ISO 14040: gestão ambiental - avaliação do ciclo de vida - princípios e estrutura", Rio de Janeiro (2009).

13. Associação Brasileira de Normas Técnicas. - "NBR ISO14044: Gestão ambiental - avaliação do ciclo de vida - requisitos e orientações", Rio de Janeiro (2009).

14. PIRA. - "PEMS 4 Manual do Utilizador", PIRA International, Leatherhead (1998).

15. Garcia, E. E. C. et al. - "Análise de ciclo de vida de embalagens para o mercado brasileiro" (1999). Relatório Final Confidencial do Projeto FAPESP.

16. Boustead, I. - "Eco-proliles of the European plastics industry - Report 2: Olefin feedstock sources", APME, Brussels (1993).

17. Associação Brasileira de Normas Técnicas. - "NBR 15792: embalagem: índice de reciclagem: definições e método de cálculo", Rio de Janeiro (2010).

Enviado: $14 / 10 / 09$

Reenviado: $29 / 03 / 10$

Aceito: $01 / 07 / 10$

DOI: $10.1590 / \mathrm{S} 0104-14282011005000003$ 